\title{
La singularité des sciences du vivant quant à la place des SHS dans les écoles d'ingénieurs en France : l'exemple de l'enseignement supérieur agronomique et agricole
}

\author{
Catherine Roby ${ }^{1}$ \\ CREAD, Université Rennes 2, Place du Recteur Paul Henry, 35000 Rennes, France
}

\begin{abstract}
Résumé. L'intégration, dans les formations d'ingénieurs, des connaissances produites par les sciences humaines et sociales (SHS) représente de forts enjeux pour les professionnels à haut niveau de qualification, notamment quant à la réflexivité nécessaire aux prises de décision en contexte de responsabilité. C'est pourquoi il semble pertinent de chercher à savoir quelle place occupent les SHS dans les formations des ingénieurs, futurs professionnels amenés à engager des choix de société. Pour aborder cette question, une enquête a cherché à identifier la place des enseignements de SHS affichés dans les curricula formels des formations initiales sous statut étudiant, présentés sur les sites internet des écoles. Quels que soient les critères utilisés (appellation générique de ces enseignements, affichage et nom des départements les regroupant ou affichage de recherches en SHS), les résultats mettent en évidence la singularité des écoles d'ingénieurs spécialisées dans les sciences de la vie et de la Terre (agronomie, biologie, géologie...). Dans le cadre de la sociologie du curriculum, cet écart est interprété comme la manifestation d'orientations culturelles technoscientifiques des écoles, pouvant être reliées à la tutelle du ministère de l'agriculture et au domaine particulier des sciences de la vie et de la Terre dans les relations qu'elles entretiennent avec les SHS.
\end{abstract}

The singularity of Life Sciences on the place of HSS in engineering schools in France: the example of agronomic and agricultural higher education

\begin{abstract}
Given the complexity of the contemporary world, the integration in Engineering Schools of knowledge produced by the Humanities and Social Sciences (HSS) represents challenges for the professionals with high level of qualification. Therefore, it seems relevant to find out which place occupy HSS in training engineers, as these future professionals will participate in societal choices. A survey was conducted to identify HSS teachings found in the formal curricula of initial training and presented on schools' websites. Regardless of the used criteria (generic name of these teachings, display and name of the department grouping them or HSS research display), the results highlight the peculiarity of Engineering Schools specialized in Earth and Life Sciences. Within the framework of the sociology of the curriculum, this gap is interpreted as the manifestation of the schools' cultural techno-scientific orientations associated with the
\end{abstract}

${ }^{1}$ Auteur de correspondance : catherine.roby@orange.fr

This is an Open Access article distributed under the terms of the Creative Commons Attribution License 4.0, which permits unrestricted use, distribution, and reproduction in any medium, provided the original work is properly cited. 
authority of the Ministry of Agriculture, and with the particular field of Life Sciences and their relation to the Humanities and Social Sciences.

Malgré les enjeux contemporains liés à la complexification des situations de travail dans le champ des sciences et des technologies (mondialisation, écologie, gestion des risques, etc.), la place des SHS dans les formations d'ingénieurs reste peu questionnée. On pourrait pourtant penser que les nombreux travaux de SHS qui ont mis en évidence les dimensions sociales et culturelles, économiques, politiques et éthiques des sciences et techniques puissent trouver leur place dans ces formations professionnelles à haut niveau de qualification. En effet, ces travaux ${ }^{2}$ lient la technique aux rapports sociaux, notamment par le biais des processus de rationalisation du travail et des organisations. Ils mettent en évidence que les savoirs scientifiques et technologiques sont le produit d'une activité humaine, dépendante d'un environnement historique et culturel et d'un contexte politique, social et économique. Que cette activité soit fortement structurée en référence à une rationalité objective, distanciée et instrumentée, ne la rend pas moins orientée par des systèmes d'intérêts et de valeurs au même titre que les autres (Brenner, 2011; Putnam, 2004) [1, 1]. Ces travaux soulèvent donc des enjeux sociétaux majeurs qui renvoient au profil scientifique et au rôle professionnel essentiel que jouent les ingénieurs pour l'avenir des sociétés technoscientifiques (Albero et Roby, 2014) [2]. En outre, le pouvoir décisionnel des ingénieurs engage souvent des choix de société pour lesquels la revendication démocratique est plus de en plus prégnante (Pestre, 2013) [4]. Dans ce contexte, les enjeux clés des SHS dans les formations d'ingénieurs concernent particulièrement la réflexivité critique sur les ressorts de l'action et peuvent être considérés au niveau des relations interdisciplinaires entre SHS et sciences et techniques de spécialité des ingénieurs (STSI). Si les SHS peuvent être instrumentalisées au titre de la rationalité de l'action humaine, elles peuvent également être en mesure d'assurer une fonction de temporisation, de prise de recul, de problématisation, face à l'accélération des changements techniques et économiques, centraux dans la formation des ingénieurs. Elles seraient ainsi à même de conduire un travail conjoint avec les STSI pour produire et diffuser de nouveaux savoirs plus adaptés aux situations professionnelles effectives (Albero et Roby, 2014 ; Roby, 2014) [2, 5].

Après une rapide présentation du contexte et des appuis théoriques de la recherche, la méthodologie ayant déjà été développée par ailleurs (Roby et Albero, 2014) [6], cette contribution ${ }^{3}$ présente dans un premier temps un résultat d'enquête empirique : la singularité du positionnement des SHS dans les écoles des sciences de la vie et de la Terre. Une revue de littérature permettra ensuite de pointer la spécificité de l'enseignement supérieur agronomique et agricole (ESA) dont relève environ la moitié de ces écoles, sous tutelle du ministère de l'agriculture ou reconnues par ce ministère. Une conclusion élargira le propos dans une perspective épistémologique.

\section{Contexte et appuis théoriques de la recherche}

Focaliser sur la place des SHS, au sens large de tous les enseignements ${ }^{4}$ qui y réfèrent ou qui en tiennent lieu dans les écoles d'ingénieurs a permis de sortir de la nébuleuse des formations qui ne relèvent pas des sciences et techniques de spécialité et dont les appellations varient considérablement. Le premier objectif de ce travail a donc été de relever le défi d'une description exhaustive des enseignements de SHS ou «faisant fonction de » puisqu'aucun état des lieux n'avait encore été produit.

\footnotetext{
${ }^{2}$ Un grand nombre de ces travaux sont référencés dans Albero et Roby (2014) [3].

${ }^{3}$ Il a été tenu compte des commentaires et des remarques faites par les relecteurs, qu'ils en soient remerciés.

${ }^{4}$ Considérées dans une acception large, comme apports susceptibles de fournir des savoirs sur l'homme et la société, philosophie, arts et lettres inclus.
} 
Ce travail doctoral (Roby, 2014) [5] s'inscrit dans un positionnement socioconstructiviste et s'appuie principalement sur la sociologie du curriculum et sur la sociohistoire des SHS et des formations d'ingénieurs. L'échelle macroscopique étant celle qui permet de repérer des stabilités culturelles (Desjeux, 2004) [7], les interrogations renvoient aux écarts potentiels de positionnement des SHS entre les écoles et à leur explication sous l'hypothèse d'une influence de différentes orientations culturelles technoscientifiques. Dans le but de repérer des particularismes et des diversités, une approche quantitative à une échelle macro-sociale, a permis de centrer l'analyse sur les curricula formels, tels qu'ils sont affichés sur les sites internet des écoles ${ }^{5}$. Cette description du paysage contemporain des SHS dans la totalité des formations initiales sous statut étudiant a porté sur leur dénomination générale, sur l'affichage des enseignements, d'un département les regroupant et de recherches en SHS.

\subsection{Sociologie du curriculum}

La sociologie du curriculum a été développée en Grande Bretagne par plusieurs sociologues dont les plus influents ont été Basil Bernstein et Michael Young au début de la décennie 1970. Considérant l'éducation, à la suite de Durkheim, comme un processus de transmission cognitive et culturelle, il s'agissait pour ces auteurs de passer d'une sociologie des institutions éducatives à une sociologie des savoirs véhiculés dans ces institutions. Les études conduites dans le cadre de cette approche sociologique portent donc sur les implications sociales des phénomènes de sélection, tri et organisation des contenus d'enseignement considérés autant sur le plan de leur symbolique que sur celui de leur organisation dans les cursus (Forquin, 2008) [8]. En étudiant les formes concrètes de cette manifestation, ce courant de recherche délimite le curriculum aux programmes d'étude tels qu'ils apparaissent dans les appellations et les modalités d'organisation des contenus d'enseignements. Les travaux mettent en valeur le caractère socialement construit du curriculum dans un processus d'élaboration permanent mobilisant des ressources humaines et matérielles dans des choix et orientations qui permettent de repérer comment se distribuent les systèmes d'intérêts et de valeurs. Le curriculum constitue ainsi un analyseur pertinent des choix qui finissent par s'instituer, définissant des territoires et des frontières, conférant des cultures et des identités.

\subsection{Notion de culture technoscientifique}

Il est désormais admis de façon consensuelle en SHS que toutes les cultures sont toujours condamnées à l'instabilité puisqu'étant en interaction, aucune n'est immuable; elles sont inscrites dans des processus de recréation perpétuelle, toujours situées et réinterprétées car constitutives d'un ensemble historique, politique, économique et social (Roby, 2014) [5]. Tout en sachant qu'il n'existe pas de déterminisme entre culture et action, le terme de "culture » est ici considéré dans le sens d'une acception «minimaliste et mesurée, pour décrire un ensemble de représentations et/ou de comportements significativement partagés par un ensemble défini d'acteurs sociaux dans un contexte donné » (Olivier de Sardan, 2008) [9].

Dans le cadre de la sociologie du curriculum, on peut considérer que les choix réalisés dans l'organisation des cursus de formation, socialement construits, relèvent de ce que l'on pourrait

\footnotetext{
${ }^{5}$ Le corpus réuni par cette étude est constitué des sites internet de 191 établissements de formation initiale d'ingénieurs sous statut d'étudiant en tant que mode de formation le plus ancien et le plus répandu (soit 93,4\% des établissements habilités par la CTI), identifiés à partir de différentes sources officielles (Journal officiel de la République Française du 10 février 2010 ; sites internet de la CTI, de la conférence des grandes écoles, du centre d'étude des formations d'ingénieurs). La liste de référence des disciplines constituant les SHS provient des sections CNU et a progressivement intégré l'ensemble des appellations utilisées par les écoles pouvant se rapporter de la façon la plus large possible à des SHS en tant que savoirs académiques sur l'humain. Des tris à plats, tris croisés, tests d'indépendance de $\chi^{2}$ avec un ensemble de variables (date de création, tutelle, domaine de spécialité, niveau de recrutement) et des analyses multivariées (analyses en composantes principales) ont été réalisés.
} 
qualifier de « culture » commune à une communauté académique et professionnelle (Forquin, 2008) [8]. Dans les écoles d'ingénieurs, la formation s'inscrit dans un ancrage historique et professionnel qui propose des formes de socialisation conduisant à l'intériorisation de modes de pensée et d'agir, de réflexion et de façons d'envisager le monde (Shinn, 1980 ; Callon, 1986 ; Thoenig, 1987 ; Belhoste, 2003 ; Lemaître, 2010) $[9,10,12,13,14]$. C'est pourquoi la notion de culture technoscientifique ou d'orientation culturelle technoscientifique des formations d'ingénieurs, comprise comme ensemble partagé de savoirs, de codes, de valeurs, de représentations, apparaît pertinente pour étudier la place et la fonction qu'y occupent les SHS. De fait, les résultats de l'enquête empirique mentionnée précédemment ont mis en évidence un positionnement singulier des écoles des sciences de la vie et de la Terre quant à la place qu'y occupent les SHS.

\section{Singularité des écoles des sciences de la vie et de la Terre}

\subsection{Des affichages différenciés}

Les affichages des appellations les plus fréquentes des enseignements de SHS, de l'existence et des noms des départements les regroupant ou encore celui de recherches en SHS, apparaissent influencés de façon significative par les dates de création des écoles, leur tutelle, niveau de recrutement et leur spécialité. En suivant Desjeux (2004) [7], ces quatre critères peuvent être considérés comme des éléments structurels stables indiquant des orientations culturelles technoscientifiques particulières des écoles, eu égard à la place accordée aux SHS dans leurs curricula.

Les résultats de l'enquête empirique mettent en évidence que dans l'ensemble des écoles, les appellations les plus fréquentes des enseignements de SHS ou qui en tiennent lieu réfèrent au mot " entreprise », alors que dans les écoles de l'ESA, ce sont les appellations "sciences sociales » et « sciences économiques et de gestion» qui sont surreprésentées. De la même façon, mais en miroir, dans les écoles spécialisées dans les sciences de la vie et de la Terre, les appellations relatives à l'entreprise, au management ou encore à la communication sont sous-représentées [5].

Par ailleurs, l'affichage de départements de SHS et encore plus celui de recherches en SHS conditionnent l'affichage des disciplines de SHS. En effet, de façon significative, les enseignements utilitaires directement liés à une activité professionnelle en entreprise sont plus affichés en absence de département et de recherches en SHS, alors que les disciplines académiques de SHS sont plus affichées lorsque des départements regroupant les enseignements de SHS et des recherches en SHS le sont également.

Les écoles généralistes, celles sous tutelle des ministères techniques et les plus anciennes, affichent significativement plus de recherches en SHS et de disciplines académiques de SHS ; c'est aussi le cas des écoles spécialisées dans les sciences de la vie et de la Terre [5]. En outre, les affichages d'un département regroupant les enseignements de SHS sont surreprésentés dans les écoles de l'ESA. Ce qui n'est pas très surprenant puisque Chosson, Martin et Veleine (1996) [15] signalaient effectivement la création de départements de SHS dans les établissements de l'ESA suite aux travaux du collectif Odyssée (voir infra) initié dans les années 1990. Cette recherche met donc en évidence une spécificité de l'ESA quant à l'affichage des SHS dans le paysage des écoles d'ingénieurs en France.

\subsection{Spécificité de l'enseignement supérieur agronomique et agricole}

En France, depuis le $18^{\mathrm{e}}$ siècle, les écoles des ministères techniques ${ }^{6}$ forment les ingénieurs des corps techniques de l'État. À ce titre, et bien qu'elles ne soient pas toutes destinées à former les plus hautes

\footnotetext{
${ }^{6}$ Sont ainsi dénommés (référence des noms en 2010) les ministères de la défense ; de l'écologie, de l'énergie, du développement durable et de la mer; de l'économie, de l'industrie et de l'emploi; de l'alimentation, de l'agriculture et de la pêche.
} 
élites du pays, elles semblent avoir conservé, dans leur ensemble, une formation concernant une assise sociale, économique et politique de la fonction d'ingénieur : capacité d'expression, ouverture d'esprit, et capacités à se situer dans un environnement politique, économique et social. Dans l'histoire de ces écoles, le développement de ces capacités passe d'abord par les humanités classiques, puis progressivement par la mobilisation des concepts des SHS, dans leur dimension réflexive ou utilitaire, voire instrumentale et potentiellement à des fins de légitimation technocratique (Roby, 2014) [5]. Bien que la formation des ingénieurs des corps techniques de l'État ne représente plus aujourd'hui qu'environ $10 \%$ des effectifs ${ }^{7}$ des écoles des ministères techniques, ces écoles restent cependant orientées par une culture technoscientifique qui les caractérise eu égard à la place qu'y occupent les SHS. Tel apparaît le cas de l'ESA, présentant, au-delà de sa diversité, une orientation spécifique.

\subsubsection{Réalité diverse et complexe de l'ESA}

Les ingénieurs du domaine de l'agriculture représentent environ $5 \%$ des ingénieurs diplômés par an, ils accèdent à des métiers variés et exercent une telle disparité de professions qu'ils ne peuvent former un groupe autonome. De fait, l'ESA recouvre une réalité complexe. La diversité des clivages, induisant une insertion professionnelle différenciée des ingénieurs, permet tout à la fois de distinguer et de regrouper les écoles. Si aujourd'hui les nouvelles concentrations tendent à estomper les écarts de positionnement, les deux grandes catégories historiquement constituées marquent néanmoins les identités locales. Il s'agit d'une part, des anciennes écoles nationales supérieures agronomiques (ENSA), plus sélectives, aux apports plus théoriques et abstraits, et d'autre part, des écoles nationales des ingénieurs des travaux agricoles (ENITA), plus récentes, aux recrutements plus ouverts et diversifiés (BTS, diplômes universitaires), aux enseignements plus appliqués. Les écoles privées sont plus territorialisées et singulièrement ouvertes aux enfants d'agriculteurs. Les filles, majoritaires dans l'ESA, sont plus nombreuses dans les ENSA que dans les ENITA et que dans les écoles privées (Charmasson, Duvigneau, Lelorrain et Le Naou, 1999) [17]. La plupart de ces ingénieurs sont malgré tout fédérés par un lien maintenu avec la référence agricole et avec le «monde agricole» (Escarboutel, 2000, p. 276) [18], lui-même intrinsèquement lié au monde vivant. Si indéniablement les mondes agricoles sont pluriels et les rapports au vivant multiples, certaines références relatives à l'humain et au monde social semblent restées inscrites dans une histoire de l'enseignement et de la recherche agronomiques, comme en attestent de nombreux travaux dans la littérature.

\subsubsection{Inscription historique et institutionnelle de la spécificité de l'ESA}

L'Institut national agronomique de Paris ouvre ses portes en 1876, il devient mixte dès 1917, juste avant l'institutionnalisation de l'enseignement féminin agricole parallèlement à l'enseignement masculin (Boulet, 1991) [19]. L'ESA a pris des formes spécifiques, notamment en termes d'ouverture sociale et sociétale et d'égalité des genres à partir des années $1960^{8}$, alors que la mixité ne s'est généralisée dans la plupart des écoles d'ingénieurs qu'après la loi Haby (1975).

Le ministère de l'agriculture, par la loi d'orientation agricole (LOA) de 1960 visant la modernisation de l'agriculture et le développement de la productivité, a porté un projet politique global intégrant la recherche, la formation et l'encadrement de la profession (technique et gestionnaire). En accord avec les représentants de la profession agricole, ce modèle s'est appuyé sur celui de l'exploitation familiale ${ }^{9}$, inscrivant l'agronomie dans un contexte politique, social et

\footnotetext{
${ }^{7}$ Il s'agit d'une moyenne nationale. L'École nationale des travaux publics d'État (environ $70 \%$ ) fait figure d'exception (Capelli, 2009) [16].

${ }^{8}$ La loi d'orientation agricole de 1960 impose à l'enseignement agricole, assuré dans des établissements féminins ou mixtes, de décerner le même diplôme pour les femmes et les hommes. La part des femmes dans l'ESA est aujourd'hui la plus importante des écoles d'ingénieurs avec un peu plus de $60 \%$.

${ }^{9}$ Il ne faudrait cependant pas oublier que cette loi a aussi introduit le mot atelier pour désigner les fonctions productives en agriculture sur le modèle tayloriste (Vissac, 2002) [24] et fait disparaître de nombreuses exploitations agricoles familiales.
} 
économique (Hubert, 2010) [20]. Cette loi précise que la réforme de l'enseignement doit prendre en considération les aspects sociaux et humains de la profession d'agriculteur. Elle prescrit également à l'enseignement agricole d'associer les personnels, les élèves, les parents et les représentants des organisations économiques (entreprises et coopératives), politiques (collectivités locales), sociales et culturelles (mouvements associatifs) concernées (Boulet, 1991) [19]. Cette politique démocratique, véritablement avant-gardiste, s'adresse aussi à $1^{\prime} \mathrm{ESA}^{10}$; cela implique des relations élargies à des organisations autres que les entreprises sur lesquelles sont concentrées la plupart des écoles d'ingénieurs. De fait, les débouchés professionnels des ingénieurs agricoles et agronomes sont moins massivement ceux des entreprises (Bonnet et Bonnet, 1997) [20].

Par ailleurs, dans les années 1970, l'ingénieur du vivant marque son identité professionnelle par son orientation culturelle technoscientifique inscrite dans une pensée systémique prenant en compte les aspects culturels, sociaux et environnementaux des exploitations agricoles; ce qui l'amène à considérer la pluralité des approches en adéquation aux différents motifs d'action des agriculteurs (Chosson, 1997 ; Dufour, 1998) [21, 23].

L'ouverture sociale et sociétale de l'enseignement agricole a été réaffirmée en 1982 au ministère de l'agriculture lorsque le directeur général de l'enseignement et de la recherche, Michel Gervais, a donné l'impulsion d'un mouvement de débat et de réflexion sans précédent dans l'histoire du système éducatif français. Il est apparu de plus nécessaire à l'enseignement agricole de tenir compte de la pluriactivité du monde rural en s'intéressant à ses divers acteurs. Ce dont atteste la réalisation d'études du milieu naturel et humain, lors des stages (à tous les niveaux de la formation agricole) qui permettent une appréhension des réalités économiques, sociales et culturelles (Boulet, 1991) [19]. La rénovation de l'enseignement agricole a consisté à mettre en évidence des objectifs généraux regroupés en modules ou en domaines pour faire comprendre à ceux qui apprennent que la formation relève à la fois de dimensions d'ordre scientifique, technique, social et culturel parce que la réalité peut être approchée sous ces divers aspects. En fait, cela n'est qu'un rappel d'une position déjà établie au ministère de l'agriculture. Dès les années 1960-1965, les mouvements d'éducation populaire ont introduit dans la notion de développement agricole une composante d'animation culturelle dans les établissements publics d'enseignement agricole. Suite à quoi, par la loi d'orientation agricole de 1966, le ministère de l'agriculture a innové par la création d'un corps d'enseignants en charge d'une éducation socioculturelle, toujours dispensée dans les lycées agricoles (Charmasson et al. 1999) [17]. La vocation de cet enseignement est de permettre le développement de la créativité et de l'initiative des élèves pour leur épanouissement personnel. L'existence de l'éducation socioculturelle a permis une ouverture sur le développement rural et agricole, dans une meilleure prise en compte de la diversité des ressources locales et des réalités humaines, sociales et culturelles au-delà des techniques et des moyens de production (Boulet, 1991) [19].

Depuis la loi d'orientation de 1984, les missions de service public de l'ESA concernent, entre autres, l'adaptation de l'enseignement agricole à son environnement économique et social, l'aménagement, la gestion, la protection de l'espace rural, de la forêt et des milieux naturels, ainsi que la coopération internationale et technique. L'ESA a également pour mission de participer à la politique de développement scientifique par les activités de recherche fondamentale et appliquée ${ }^{11}$ (ibid.).

La loi d'orientation de 1999 prône une agriculture française et européenne multifonctionnelle et citoyenne. Elle appelle à l'évolution de l'enseignement agricole par la prise en compte des fonctions économique, environnementale et sociale de l'agriculture ainsi que par la participation à l'aménagement du territoire en vue d'un développement durable dans toutes les composantes de l'enseignement agricole (secondaire, supérieur court, supérieur long, apprentissage, formation continue et formation ouverte et à distance) (Escarboutel, 2000) [18]. Dans ce contexte, le développement durable a été intégré aux enseignements dans les établissements scolaires du ministère de l'agriculture dès le début des années 2000, Laurence et Jean Simonneaux (2014) [25] ayant

\footnotetext{
${ }^{10}$ Le réseau de l'ESA intègre les établissements privés sous contrat avec l'État (ministère de l'agriculture).

${ }^{11}$ L'enseignement supérieur agricole privé a les mêmes missions que l'enseignement public.
} 
d'ailleurs conduit des travaux pionniers de recherche en didactique sur les questions socialement vives.

Manifestement, l'enseignement agricole a été porté dans les années 1960 et 1970 par un projet politique humaniste qui a également touché l'ESA. Si la place de l'État dans la recherche, la formation, l'appui technique aux exploitants agricoles, demeure importante (Hubert, 2010) [20], il resterait à étudier aujourd'hui comment la dimension idéelle (Albero, 2010) [26] des différents dispositifs de formation en place dans les écoles de l'ESA s'est transformée depuis les années 1980.

\subsection{Inscription des SHS dans la spécificité de l'ESA}

Dans la majeure partie des écoles de l'ESA, les SHS sont attestées de longue date comme connaissances de base, obligatoires et incluses dans un tronc commun, impliquées dans les travaux de terrain des élèves-ingénieurs (Hubert, 2010 ; Dufour, 1998 ; Cépède et Madec, 1966 ; Cheïko, 2002) $[20,23,26,28]$. Ces dimensions de la formation sont probablement à relier à l'importance du développement précoce de la sociologie rurale en France. En effet, le groupe de sociologie rurale créé par Lefebvre et Mendras en 1950 est celui qui a le plus bénéficié des contrats et crédits de la Direction générale de la recherche scientifique et technologique (DGRST) dans les années 1960 ; il comptait 25 chercheurs en 1965 (Masson, 2006) [28]. Il n'est donc pas étonnant que le mouvement de l'ouverture sociale et sociétale des écoles de l'ESA, dès les années 1960, se soit accompagné du développement des formations en SHS (Chosson, 1997) [21]. Par ailleurs, même si les fonctions politiques du sociologue Bertrand Hervieu peuvent être considérées comme le fruit d'une trajectoire personnelle, on peut penser que leur inscription dans une longue durée pourrait avoir conduit quelques responsables du ministère de l'agriculture à une certaine familiarisation avec les sciences sociales ${ }^{12}$.

Au début des années 1990, l'ESA s'est inscrit dans la dynamique de réflexion sur la place des humanités dans la formation des étudiants lancée par la Conférence des grandes écoles. Le collectif «Odyssée », groupe d'enseignants-chercheurs de SHS de 1'ESA ${ }^{13}$ a été constitué pour conduire une réflexion collective sur la formation humaine et culturelle (Chosson, 1996) [29]. Il visait à promouvoir la place des SHS dans les écoles qui n'étaient pas encore toutes préparées à les recevoir ; ces enseignements n'ayant pas toujours trouvé leur place ni gagné leur légitimité (Laquièze, 2009) [30]. Les membres de ce collectif considèrent comme anti-modèle la tendance techniciste parée des «atours d'une modernité scientifique» de certaines formations aux humanités ou en SHS dans les grandes écoles (Chosson et al., 1996) [15]. Dans cette tendance mécaniste, des outils sont présentés comme des clés d'action sur les comportements des individus, censés les rendre "plus performants, plus créatifs, plus aptes à mobiliser les hommes au service du projet d'entreprise » (ibid.). Cette approche empruntant des concepts de différentes disciplines de SHS, telles que la psycho-sociologie, la psychologie cognitive, la sociologie des organisations, est pour eux éloignée des réels contenus disciplinaires des SHS. Ils placent la philosophie au centre du débat sur les valeurs et plaident pour l'enseignement des méthodes des sciences sociales dans les formations des grandes écoles.

\footnotetext{
${ }^{12}$ Bertrand Hervieu a été chargé de la sous-direction de la recherche et des études au ministère de l'agriculture (1982-1985), conseiller technique du ministre de l'agriculture, puis directeur général de l'enseignement et de la recherche (1985-1986), conseiller technique du Premier ministre sur les questions rurales (1991-1992), chargé du développement rural, des forêts, de l'enseignement et de la recherche, conseiller technique du ministre de l'agriculture et de la pêche, chargé de la préparation de la loi d'orientation agricole et de la loi d'orientation forestière (1997-1998), conseiller du ministre de l'agriculture et de la pêche (1998-1999), puis élu à la présidence de l'INRA où il siégea de 1999 à 2003. Bertrand Hervieu a été nommé vice-président du Conseil général de l'alimentation, de l'agriculture et des espaces ruraux par arrêté du 25 juillet 2012 et par Stéphane Le Foll, Ministre de l'agriculture, de l'agroalimentaire et de la forêt. http://fr.wikipedia.org/wiki/Bertrand_Hervieu, et http://agriculture.gouv.fr/Bertrand-HERVIEU-est-nomme-Vice, consultés le 2 octobre 2013.

${ }^{13}$ Regroupés dans la section 9 de la Commission nationale des enseignants chercheurs relevant du ministère de l'agriculture (CNECA) dénommée « Sciences économiques, sociales et humaines » les enseignants chercheurs de SHS représentent environ $13 \%$ des neuf sections principales de la CNECA (Veleine et Bargeot, 2009) [35].
} 
Par ailleurs, Lacombe (2009) [31] précise qu'enseignement et recherche dans les sciences sociales (économie rurale et sociologie rurale) ont largement été associés dans l'ESA.

D'autres chercheurs en SHS de l'ESA (Bonnet et Bonnet, 1997 ; Dufour, 1998) [20, 23] proposent comme objectifs stratégiques de formation des ingénieurs des techniques agricoles le développement de compétences d'analyse des situations complexes par des capacités d'articulation entre des aptitudes pratiques, des savoirs techniques, sociaux et culturels, perçus comme imbriqués et complémentaires. Ces capacités d'analyses doivent aider à la résolution pertinente des problèmes complexes par le dépassement du cloisonnement de différents constats, tout comme peut le faire également la capacité de croisement et de mise en interaction de différents modèles d'analyse, de pensée et d'action. Ces objectifs conduisent à envisager la formation sur la base d'interactions entre les objets et les méthodes des champs disciplinaires. La compréhension des mécanismes de communication sociale et interculturelle doit permettre d'appréhender les différents modes de réflexion et les logiques d'action correspondantes dans les sphères professionnelles. Il s'agit d'intégrer dans les décisions, les évolutions et les adaptations possibles des solutions proposées (Bonnet et Bonnet, 1997) [20]. Sylvestre (2009) [33] rend compte également d'un souci de former des ingénieurs agronomes et agricoles réflexifs, en cherchant à développer des capacités d'approche de la complexité par un enseignement sur la culture d'entreprise, inscrite dans des règles sur les échanges économiques et sociaux. De même, la formation des ingénieurs forestiers intègre la prise en compte des spécificités locales et historiques de chaque forêt, les différentes perceptions humaines et les différents usages sociaux de la forêt, le raisonnement à long terme. Dès lors, il est acquis qu'enseigner une forêt objective ne peut relever que de l'illusion (Cheïko, 2002) [28].

Par ailleurs, l'enquête sur la formation humaine des ingénieurs, conduite au milieu des années 1990 par Giré, Béraud et Déchamps (2000) [34], mettait en évidence la position atypique de l'Institut National Agronomique Paris Grignon (INAPG). Dans cette école, la formation humaine paraissait suffisante ou assez suffisante pour $47 \%$ des élèves-ingénieurs et ingénieurs interrogés, alors que ce n'était le cas que pour $37 \%$ des élèves et ingénieurs des Ponts et chaussées, et pour $32 \%$ de ceux des Arts et métiers. Même si on peut s'interroger sur ce qui apparaît comme "suffisant» aux yeux des uns et des autres, cet écart semble congruent avec les éléments de la littérature sur les enseignements de SHS dans l'ESA. En outre, Escarboutel (2000) [18] a montré que les étudiants des deux écoles de l'ESA de Toulouse choisissent leur formation en raison de leur attrait pour la biologie et la nature. Ils sont majoritairement en désaccord avec l'idée d'un choix d'études qui résulterait d'une volonté de rémunération élevée et sont caractérisés par une forte volonté d'autonomie personnelle. Ils considèrent majoritairement, et les femmes encore plus, que la communication et la sociologie sont de véritables outils professionnels qu'ils apprécient favorablement comme tels. De fait, les sciences agronomiques sont enracinées autant dans des pratiques sociales que dans des connaissances théoriques. Étudier la parcelle agricole nécessite pour les chercheurs de prendre en compte les pratiques locales et sociales de l'agriculteur ainsi que les activités humaines de son environnement tout autant que les politiques agricoles ${ }^{14}$ (Dufour, 1998; Vissac, 2004 ; Cheïko, 2002 ; Bonneuil, Denis et Mayaud, 2008) [23, 24, 28, 36]. L'agronomie, composante historique des formations d'ingénieurs de l'ESA, présente effectivement un caractère scientifique systémique exigeant une intégration interdisciplinaire, liée à sa finalité professionnelle. D'ailleurs la grande majorité des écoles de l'ESA, qu'elles soient sous tutelle du ministère de l'agriculture ou non, accueille des laboratoires de recherche en SHS ou intégrant des SHS, souvent dans le cadre d'unités mixtes de recherche

\footnotetext{
${ }^{14}$ Selon le rapport Canepa (2009, p. 16) [39], $85 \%$ des ingénieurs du corps du génie rural, des eaux et des forêts (GREF) ne sont pas issus de Polytechnique. Cela aurait-il une incidence sur les conceptions des formations dans les établissements de l'ESA ? Les membres du GREF étant principalement issus de l'Institut national agronomique de Paris Grignon (AgroParis Tech aujourd'hui), il se pourrait que les enseignements des sciences du vivant et ceux de sociologie aient pu donner à ces ingénieurs une moindre illusion de maîtrise, une ouverture à l'incertitude et une disponibilité plus importante aux réalités sociales.
} 
(UMR) impliquant un organisme tel que l'INRA, l'IRD, le CIRAD ou l'IRSTEA ${ }^{15}$ et articulant des sciences et techniques de spécialité à des SHS (Prévost et Martinand, 2012) [37]. Or, les résultats de la recherche mentionnée supra montrent un lien significatif entre l'affichage des recherches en SHS dans les écoles et celui des enseignements en SHS (Roby, 2014) [5]. La mobilisation des SHS, incontournable dans les recherches en agronomie et en sciences de l'environnement (Hubert, 2010 ; Létourneau, 2010) [20,37], semble donc bien une singularité des sciences agronomiques, face au développement d'une agriculture multifonctionnelle et aux nécessités du développement durable.

\subsection{La singularité des sciences agronomiques}

Pour rendre compte de la spécificité de l'orientation culturelle technoscientifique des écoles d'ingénieurs de l'ESA, la référence aux sciences agronomiques dans leur faculté de dialogue avec les SHS semble donc pertinente. Les exemples de trois instituts de recherche peuvent illustrer ce positionnement particulier: l'IRD, le CIRAD et l'INRA $^{16}$. Dans ces instituts où la recherche agronomique tient une place essentielle :

la sociologie et l'économie agricole sont des disciplines mobilisées depuis longtemps aux côtés de l'agronomie pour le traitement des questions spécialisées de l'activité agricole, ensuite sont venues les sciences politiques et le droit pour élargir l'analyse des activités avec la prise en compte des questions d'aménagement du territoire puis environnementales (Hubert, 2010) [20].

\subsubsection{L'INRA et l'interdisciplinarité}

À la fin des années 1960, des chercheurs agronomes de l'INRA se sont interrogés sur les résistances du monde agricole à la modernisation ${ }^{17}$. Dès 1970 , leurs recherches, menées dans différentes régions de France, leur ont permis de comprendre que les exploitants qui ne suivaient pas les conseils du développement agricole n'étaient pas des «attardés » mais des homo faber qui avaient de bonnes raisons de faire ce qu'ils faisaient là où ils étaient. Leurs pratiques indigènes, reliées à leur histoire, à leur situation, à leur identité personnelle, professionnelle et sociale, étaient ancrée dans un territoire, alors que les techniques ressortaient d'une conception exogène, inscrite dans une politique agricole. Les résistances endogènes des éleveurs de certaines régions françaises aux modèles généraux du développement agricole ont appris aux agronomes les limites de leurs conceptions théoriques. Ces derniers ont perçu que leurs innovations techniques devaient répondre à des besoins ressentis et ne pas contrarier les systèmes de valeurs et de pouvoirs des agriculteurs. Ils ont appris et compris que la conduite du développement nécessitait autant une validation sociale que technique de leurs propositions, par le biais d'une recherche-action confrontant les savoirs locaux aux savoirs érudits (Vissac, 2002) [24]. Ces constats sur l'importance des SHS dans l'analyse et l'organisation des activités agricoles sont d'autant plus pertinents aujourd'hui, dans la mesure où :

Une vaste expertise collective sur les questions agricoles mondiales met en évidence les quelques impasses auxquelles peuvent conduire des choix trop étroits en matière d'options technologiques, l'ignorance des savoirs locaux, la négligence du rôle des femmes, une vision étroite des droits de propriété intellectuelle, etc. (Hubert, 2010) [20].

\footnotetext{
${ }^{15}$ INRA, Institut national de la recherche agronomique ; IRD, Institut de la recherche pour le développement ; CIRAD, Centre de coopération internationale en recherche agronomique pour le développement; IRSTEA, Institut national de recherches en sciences et technologies pour l'environnement et l'agriculture.

${ }^{16}$ L'INRA et l'IRD ont d'ailleurs rejoint l'alliance Athena (Alliance nationale des SHS) en 2014.

${ }^{17}$ Seul un tiers des agriculteurs suivait les conseils techniques (Vissac, 2002) [24].
} 
Les apports des SHS de ces instituts ${ }^{18}$ ont conduit à l'évidence de la nécessité de changer le paradigme unidimensionnel de la pensée agronomique, fondé sur «l'évidence » de la productivité, pour envisager de nouveaux cadres théoriques et repères (diversification, extensification, multifonctionnalité, intensification écologique). En prenant conscience que les choix technologiques sont aussi des choix sociaux, environnementaux, économiques et politiques ${ }^{19}$, il s'agit maintenant, dans une vision sociale et sociétale du développement, de concevoir une gestion des ressources naturelles renouvelables. Cette gestion vise à hybrider les savoirs en mobilisant autant les méthodes et les concepts de l'agronomie, de l'écologie et des sciences sociales que les pratiques et savoirs locaux. Ceci, afin de laisser émerger une diversité de modèles agricoles, créateurs de différentes formes d'environnement et adaptés aux types de production, situations géographiques, modes de soutien politique, engagements et valeurs individuels et collectifs (Hubert, 2010) [20]. Il devient donc admis par certains chercheurs de l'INRA que les savoirs produits par les scientifiques doivent prendre place parmi une pluralité de systèmes de pensée et une diversité de logiques (Vissac, 2004) [24].

Sous l'impulsion de la DGRST ${ }^{20}$ et avec son soutien financier, l'INRA a créé le département Sciences pour l'action et le développement (SAD) en 1979 dont la mission a été de promouvoir l'interdisciplinarité. Ses travaux privilégient «l'analyse conjointe des projets et des actions qui relient les faits de nature et les faits de société, la formalisation des cohérences entre ces différentes catégories de faits » (Hubert et Bonnemaire, 2000) [39]. Il est constitué de chercheurs en agronomie, zootechnie, écologie, économie, sociologie, gestion, anthropologie, géographie, sciences cognitives. Son premier directeur, Bertrand Vissac, témoigne de son expérience de la pratique de l'interdisciplinarité qui n'avait pas encore vraiment de légitimité dans les années 1970, alors que dans les années 1950, le CNRS avait initié des actions thématiques dont l'une, la recherche coopérative sur programme, a concerné les éleveurs de l'Aubrac. Elle a fait collaborer des ethnologues et sociologues du CNRS avec des zootechniciens de l'INRA. La technique de l'élevage a alors été appréhendée comme un fait social collectif et la confrontation des points de vue des ethnologues et des sociologues a montré la richesse de "l'information qualitative, seule susceptible de saisir la complexité du fonctionnement des élevages » (Vissac, 2004, p. 288) [24].

De fait, à la suite de l'agriculture, l'agronomie est inscrite dans un contexte politique, économique et social nécessitant une approche interdisciplinaire entre les sciences de la nature, dont celles du vivant et les $\mathrm{SHS}^{21}$. C'est également ce dont témoignent d'autres instituts de recherche de ce domaine dont sont généralement proches quelques établissements de l'ESA.

\subsubsection{L'IRD et le CIRAD}

L'IRD a pour objet de contribuer au développement social, économique et culturel des pays du sud, il privilégie l'interdisciplinarité dans ses recherches sur les relations entre l'homme et son environnement $^{22}$. En 2002, les chercheurs en SHS de l'IRD représentaient environ $8 \%$ du total des

\footnotetext{
${ }^{18}$ Certes toutes les prises de conscience des problèmes posés par l'intensification, la modernisation et la rationalisation dans les productions agricoles et agroalimentaires ne viennent pas uniquement de ces instituts de recherche, mais l'INRA intègre les travaux qui pointent les méfaits de l'idéologie productiviste (Bonneuil et al., 2008) [36]. Généticien à l'INRA, Bernard Vissac a analysé la loi de 1966 sur l'élevage comme la mise en place d'une organisation sociotechnique et comme une « véritable révolution sociologique silencieuse » (Visssac, 2004, p. 195) [24]. Il reconnaît l'importance des SHS, tout autant que leur côté subversif expliquant qu'elles aient été «mobilisées aux marges, et en tout cas, non sollicitées par les politiques dont elles auraient pu contrarier la volonté de modernisme » (ibid., p. 197) [24].

${ }^{19}$ Comme en témoignent les conférences «Sciences en question» de l'INRA initiées en 1994 à l'initiative du service formation de la Direction des ressources humaines.

${ }^{20}$ On peut souligner l'importance des politiques publiques de recherche pour le développement des SHS (Roby, 2014) [5].

${ }^{21}$ Georges Guille-Escuret confirme cette interdisciplinarité à 1'INRA, «si rare ailleurs»: http://www.canalu.tv/video/centre_d_enseignement_multimedia_universitaire_c_e_m_u/la_malediction_epistemologique_sur_les

sciences_sociales_et_1_impossible_interdisciplinarite.13031 $(\overline{1} . \overline{01} . \overline{58})$

${ }^{22} \mathrm{http}: / /$ www.ird.fr/, consulté le 14 mai 2013.
} 
chercheurs en $\mathrm{SHS}^{23}$ présents dans les établissements publics à caractère scientifique et technique (EPST) alors que les chercheurs en SHS de l'INRA et du CEMAGREF ${ }^{24}$ représentaient environ $7 \%$ $\mathrm{du}$ total des chercheurs en SHS de ces établissements (Godelier, 2002) [41]. Le CIRAD a pour mission d'accompagner les pays du sud dans leur développement agricole. Ses activités relèvent « des sciences du vivant, des sciences sociales et des sciences de l'ingénieur appliquées à l'agriculture, à l'alimentation et aux territoires ruraux ${ }^{25}$. Ses effectifs de chercheurs en SHS représentaient $22 \%$ des chercheurs en $\mathrm{SHS}^{26}$ des établissements publics à caractère industriel et commercial (EPIC) en 2002. $\mathrm{Si}$ on considère ces quatre organismes de recherche scientifiques, IRD, CIRAD, INRA, IRSTEA, dont les activités sont liées aux sciences du vivant et de l'environnement, leurs effectifs de chercheurs en SHS représentaient $16 \%$ des effectifs de chercheurs en SHS des EPST et des EPIC en 2002 (ibid.). Ces organismes de recherche soutiennent aujourd'hui, aux côtés de l'IFREMER ${ }^{27}$ et du CNRS, la revue interdisciplinaire ${ }^{28}$ Nature, science, société créée en 1993, ce qui rend compte de la dynamique de l'approche interdisciplinaire dans les domaines des sciences du vivant. Les publications des éditions QUAE (CIRAD, IFREMER, INRA, IRSTEA) témoignent d'une approche résolument ouverte sur les savoirs ${ }^{29}$. Il n'est donc pas surprenant qu'apparaissent dans certaines formations supérieures d'ingénieurs sous tutelle du ministère de l'agriculture, ainsi que dans des écoles qui relèvent du domaine de spécialité des sciences de la vie et de la Terre, une pluridisciplinarité incluant les SHS, voire parfois une certaine tendance vers l'interdisciplinarité (Dufour, 1998; Cheïko, 2002 ; Doré, 2009 ; Camel et Fargue-Lelièvre, 2009) [23, 28, 41, 43]. Les acteurs de ces formations auraientils, plus que d'autres, une intuition de la richesse potentielle de l'hybridation des savoirs ou de leur nécessaire complémentarité ?

\section{3 Éléments de conclusion}

La description du panorama des SHS dans les formations d'ingénieurs en France, à partir de leur affichage dans les présentations des curricula formels sur les sites internet des écoles, a permis de mettre en évidence la singularité des écoles spécialisées dans les sciences de la vie et de la Terre. Elles affichent significativement plus d'enseignements, de département les regroupant et de recherches en SHS, marquant ainsi des orientations culturelles technoscientifiques différenciées des autres écoles (Roby, 2014) [5].

D'autre part, la place spécifique avérée des SHS dans 1'ESA formant des ingénieurs spécialisés dans les sciences du vivant permet de la mettre en lien avec trois facteurs principaux :

- Le développement important de la sociologie rurale dans les décennies 1950 et 1960 durant lesquelles se met en place l'institutionnalisation des SHS en France,

- La mission de recherche en SHS explicitement confiée aux établissements de l'ESA par la LOA de 1984,

- L'implication des enseignants-chercheurs de SHS de l'ESA dans la dynamique de renouveau des réflexions sur les enseignements de SHS, impulsée dans les formations d'ingénieurs dans les années 1990.

\footnotetext{
${ }^{23}$ Plein temps.

${ }^{24}$ Centre d'étude du machisme agricole, du génie rural et des eaux et forêts, né en 1981 de la fusion de deux organismes de recherche technique du ministère de l'agriculture, il a pris en 2011 un nom qui rend compte de l'évolution de ses activités dans le domaine agro-environnemental, IRSTEA (voir supra).

${ }^{25} \mathrm{http}: / / w w w . c i r a d . f r / q u i-s o m m e s-n o u s / l e-c i r a d-e n-b r e f$, consulté le 14 mai 2013.

${ }^{26}$ Plein temps.

${ }^{27}$ Institut français de recherche pour l'exploitation de la mer.

${ }^{28} \mathrm{La}$ revue a pour vocation d'accueillir tout texte original traitant des interactions entre les sociétés et leur environnement. http://www.nss-journal.org et http://www.nss-dialogues.fr/Objectifs, consultés le 2 octobre 2013.

${ }^{29}$ Notamment la collection Indisciplines qui accueille des ouvrages pluridisciplinaires traitant des rapports que l'homme entretient avec la nature, et les collections Nature sociale et sciences en question. http://www.quae.com/, consulté le 2 octobre 2013.
} 
Ces éléments indiquent des orientations culturelles technoscientifiques particulières pour les ingénieries agricoles, plus ou moins amenées à penser la technique constitutive de pratiques humaines contextualisées dans une histoire sociale et politique. Cependant, même si les sciences du vivant et les SHS partagent une dimension culturelle (Chosson, 1997) [21] et bien que les SHS tendent à habiter la culture technoscientifique, spécifique à cet égard, de la plupart des écoles de l'ESA, beaucoup reste à faire pour découvrir l'originalité et la fertilité des SHS (Vincent, 1996) [44].

Certes, ni les sciences du vivant ni les SHS ne représentent des mondes homogènes et les clivages épistémologiques ne traversent pas des groupes de disciplines, quels qu'ils soient, mais bien chacune des disciplines. Cependant, à une macro échelle et dans une longue temporalité, quelques éléments d'ordre sociohistorique et épistémologique rapprochant sciences naturelles (ou sciences de la vie et de la Terre aujourd'hui) et SHS méritent d'être rappelés :

- À la fin du $19^{\mathrm{e}}$ siècle, les sciences naturelles et les SHS ont eu à faire la preuve de leur scientificité et à lutter contre l'ordre religieux établi (Blanckaërt, 2006) [45] ;

- L'observation, l'induction et le dialogue caractérisent l'enseignement dans les sciences de la vie et de la Terre dont les contenus sont proches de la science en train de se faire et fréquemment reliés à des questions socialement vives (Hulin, 2014; Tiberghein, 2009) [46, 47], ce qui les rapproche des SHS ;

- La plupart des sciences de la vie et de la Terre et des SHS rencontrent des difficultés de généralisation; elles sont souvent face à l'impossibilité des expérimentations «toutes choses égales par ailleurs » et doivent tenir compte du rôle des interactions, ce qui les conduit potentiellement à des approches holistiques, systémiques, et couramment au renoncement d'une maîtrise totale des phénomènes, qu'ils soient expérimentaux ou non.

Ces particularités épistémologiques pourraient entraîner par exemple une considération différente des normes et procédures organisationnelles et de management pour des ingénieurs formés par les sciences physiques dans les sciences industrielles et ceux formés par les sciences de la vie et de la Terre. Les formations peuvent effectivement induire des visions du monde particulières : Canguilhem (1952/2009) [48] rappelait que pour les ingénieurs, les hérissons traversent les routes, alors que pour un écologue, les hérissons explorent leur milieu en fonction de leurs impulsions alimentaires et sexuelles : cette compréhension du vivant n'est peut-être pas forcément accessible de la même façon à tous les ingénieurs.

Par ailleurs, le questionnement philosophique et épistémologique semble récurrent au cœur même des sciences du vivant comme en témoigne le colloque relaté par ces actes. En effet, il paraît de plus en plus difficile aujourd'hui d'occulter la portée sociale et politique, voire anthropologique, des recherches et des manipulations techniques sur le vivant, alors que l'apolitisme des ingénieurs dans les domaines industriels reste affirmé face à une science industrielle et à une technique, encore généralement prétendues neutres (Roby, 2014) [5].

C'est peut-être pourquoi, les écoles des sciences de la vie et de la Terre apparaissent comme une singularité dans le paysage des écoles d'ingénieurs en France, eu égard à la place qu'y occupent les SHS. Elles pourraient rendre compte de quelques engagements notoires dans de rares écoles où les SHS tendent à être intégrées (plus ou moins en interdisciplinarité) alors qu'il existe encore peu de SHS en tant que disciplines scientifiques dans les écoles d'ingénieurs (condition sine qua non à l'interdisciplinarité). Cette intégration pourrait faciliter une compréhension sociotechnique des situations d'ingénierie, considérées dans leur dimension humaine, c'est à dire vivante ; une dimension dont les formes ne peuvent être saisies que dans une vision, jamais dans une division (Canguilhem, 1952/2009) [48].

\section{Références}

1. Brenner, Raison scientifique et valeurs humaines : essai sur les critères du choix objectif (PUF, Paris, 2011). 
2. H. Putnam, Fait-valeur : la fin d'un dogme et autres essais (Éclat, Paris, 2004).

3. B. Albero, C. Roby, Les enjeux du rapport aux sciences humaines et sociales dans la formation des ingénieurs en France, Revue française de pédagogie, 186, 59-73 (2014).

4. D. Pestre, À contre-science: politiques et savoirs des sociétés contemporaines (Seuil, Paris, 2013).

5. C. Roby, Place et fonction des SHS dans les écoles d'ingénieurs en France : état des lieux, enjeux et perspectives épistémologiques (Thèse, Université Rennes 2, 2014).

6. C. Roby, B. Albero, Les sciences humaines dans les parcours scientifiques et techniques professionnalisants: quelles finalités et quelles modalités pratiques? 1 (Société française d'histoire des sciences et des techniques, Paris, 2014).

7. D. Desjeux, Les sciences sociales (PUF, Paris, 2004).

8. J. C. Forquin, Sociologie du curriculum (PUR, Rennes, 2008).

9. J. P. Olivier de Sardan, La rigueur du qualitatif : les contraintes empiriques de l'interprétation socio-anthropologique, 33 (Academia-Bruylant, Louvain-La-Neuve, 2008).

10. T. Shinn, Division du savoir et spécificité organisationnelle. Les laboratoires de recherche industrielle en France, Revue française de sociologie, 21(1), 3-35 (1980).

11. M. Callon, Eléments pour une sociologie de la traduction. La domestication des coquilles SaintJacques et des marins-pêcheurs dans la baie de Saint-Brieuc. L'année sociologique, 36, 169-208 (1986).

12. J. C. Thoenig, L'ère des technocrates : le cas des Ponts et chaussées (L'Harmattan, Paris, 1987)

13. B. Belhoste, La formation d'une technocratie : l'École polytechnique et ses élèves de la Révolution au Second Empire (Belin, Paris, 2003).

14. D. Lemaître, Curriculum, pratiques pédagogiques et modèles de formation : le cas des grandes Écoles en France (HDR, Université de Nantes, 2010).

15. J. F. Chosson, F. Martin, C. Véleine, POUR, 151, 13 (1996.).

16. F. Capelli, La socialisation de l'élite scolaire : une ethnographie du parcours d'apprentissage des jeunes d'une grande école d'ingénieurs française (Thèse, Université Grenoble 2, 2009).

17. T. Charmasson, M., Duvigneau, A.-M. Lelorrain, H. Le Naou, L'Enseignement agricole, 150 ans d'histoire, évolution historique et atlas contemporain (Educagri, Dijon, 1999).

18. M. Escarboutel, Itinéraires professionnels et identité des ingénieurs issus du système d'enseignement supérieur agricole : le cas des ingénieurs agronomes de l'ENSAT et des ingénieurs en agriculture de l'ESAP (Thèse, Université Toulouse 2, 2000).

19. M. Boulet, De l'enseignement agricole au savoir vert (L'Harmattan, Paris, 1991).

20. B. Hubert, L'agronomie, science de l'agriculture ? Le Mouvement Social, 233, 143-157 (2010).

21. R. Bonnet, J. Bonnet, Ingénieurs des techniques agricoles : vers de nouvelles relations formationemploi. L'orientation scolaire et professionnelle, 26(3), 335-350 (1997).

22. J. F. Chosson, L'ingénieur du vivant: un intermédiaire culturel, Économie et humanisme, 340, 40-45 (1997).

23. A. Dufour, Les enjeux de l'enseignement de la sociologie dans une École d'ingénieur. Analyse du curriculum de l'Institut supérieur d'agriculture Rhône-Alpes de 1968 à 1994 (Thèse, Université Lyon 2, 1998).

24. B. Vissac, Les vaches de la République : saisons et raisons d'un chercheur citoyen (INRA Éditions, Paris, 2002).

25. L. Simonneaux, J. Simonneaux, Panorama de recherches autour de l'enseignement-apprentissage des Questions Socialement Vives, Revue francophone du Développement durable, 4, 109-126 (2014). 
26. B. Albero, Enjeux et dilemmes de l'autonomie. Une expérience d'autoformation à l'université. Étude de cas, 67 (MSH Éditions, Paris, 2010).

27. M. Cépède et A. Madec, Contribution de la sociologie à l'économie rurale, Économie rurale, 70(1), 3-14 (1966).

28. M. Cheiko, Pluridisciplinarité et foresterie : recherche, gestion, pédagogie de projet et formation des ingénieurs forestiers (Thèse, Université Lyon 1, 2002).

29. P. Masson, Le financement de la sociologie française : les conventions de recherche de la DGRST dans les années soixante, Genèses, 62, 110-128 (2006).

30. J. F. Chosson, La formation humaine dans l'enseignement supérieur agronomique et vétérinaire Un patrimoine - un concept-horizon - des pratiques plurielles, POUR, 151, 7-9 (1996).

31. B. Laquièze, Enseignement supérieur agricole, formation des cadres et expression citoyenne, POUR, 200, 93-102 (2009).

32. P. Lacombe, Formation des agronomes et sciences sociales, POUR, 200, 119-131 (2009).

33. J. P. Sylvestre, Identité professionnelle et culture d'entreprise, POUR, 151, 129-141 (1996).

34. A. Giré, A. Béraud, P. Déchamps, Les ingénieurs : identités en questions (L'Harmattan, Paris, 2000)

35. C. Veleine, M. Bargeot, Les femmes et les hommes dans les écoles publiques de l'enseignement supérieur agronomique et vétérinaire, POUR, 200, 143-149 (2009).

36. C. Bonneuil, G. Denis, J. L Mayaud, Sciences, chercheurs et agriculture. Pour une histoire de la recherche agronomique (Quae - L'Harmattan, Paris, 2008).

37. P. Prévost, J. L. Martinand, XVIIème Congrès de l'Association mondiale des sciences de l'éducation, AMSE : enjeux et défis aujourd'hui (Reims, 2012) http://www.lirdef.univmontp2.fr/node/3132012

38. A. Létourneau, VertigO, La transdisciplinarité considérée en général et en sciences de l'environnement, La revue électronique en sciences de l'environnement, 8(2) (2010)

39. D. Canepa, J. M. Folz, F. Blazy, Mission d'étude sur l'avenir des corps d'ingénieurs de l'État. (Rapport remis au Premier Ministre, Paris, 2009).

40. B. Hubert, J. Bonnemaire, La construction des objets dans la recherche interdisciplinaire finalisée : de nouvelles exigences pour l'évaluation, Natures Sciences Sociétés, 8(3), 5-19 (2000).

41. M. Godelier, L'état des Sciences de l'Homme et de la Société en France et leur rôle dans la construction de l'Espace Européen de la Recherche (Rapport remis au Premier Ministre, Paris, 2002).

42. T. Doré, L'évolution de l'enseignement de l'agronomie à l'INA Paris-Grignon face aux changements sociétaux, scientifiques et institutionnels, POUR, 200, 111-118 (2009).

43. V. Camel, A. Fargue-Lelièvre, Analyse de pratiques interdisciplinaires dans l'enseignement supérieur, Revue internationale de pédagogie de l'enseignement supérieur, 2(25), 1-17 (2009).

44. J. Vincent, Culture scientifique humanisée, pédagogies actives et autoformation à l'INAP P-G, POUR, 151, 39-53 (1996).

45. C. Blanckaert, Qu'est-ce qu'une discipline ? 117 (EHESS Éditions, Paris, 2006).

46. N. Hulin, Les sciences naturelles: histoire d'une discipline du XIXe au XXe siècle (L'Harmattan, Paris, 2014).

47. A. Thiberghien, Enseigner des controverses, 1 (PUR, Rennes, 2009).

48. G. Canguilhem, La connaissance de la vie. Deuxième édition revue et augmentée (Vrin, Paris, 1952/2009). 